\title{
Response of Hinode XRT to quiet Sun, active region and flare plasma
}

\author{
B. O’Dwyer, G. Del Zanna, and H. E. Mason
}

\begin{abstract}
Department of Applied Mathematics and Theoretical Physics, Wilberforce Road, Cambridge CB3 0WA, UK e-mail: [B.0-Dwyer; G.Del-Zanna; H.E.Mason]@damtp.cam.ac.uk
\end{abstract}

Received 17 December 2010 / Accepted 28 October 2013

\begin{abstract}
Aims. We examine the response of the Hinode X-Ray Telescope (XRT), using simultaneous observations with the Hinode Extremeultraviolet Imaging Spectrometer (EIS), for a flare, an active region and a quiet Sun region. We also examine the relative intensity calibration of EIS and XRT.

Methods. EIS differential emission measure distribution (DEM) curves were used to create synthetic spectra with the CHIANTI atomic database. The contribution of spectral lines and continuum emission to each of the XRT channels was determined from the synthetic spectra, which were then convolved with the effective area of each XRT channel. The predicted total count rates for each channel were compared with the observed count rates. The effects of varying elemental abundances and the temperature range for the inversion were investigated. DEMs obtained from the XRT bands were also computed and compared to those obtained with EIS. Results. For the active region observations, the observed XRT count rates for most of the channels are in reasonable agreement with those predicted using EIS observations, but are dependent on the elemental abundances chosen. Significant discrepancies between predicted and observed count rates were found and are discussed for the adjacent quiet Sun region and also for the flare. Synthetic spectra and continuum emission contributing to the XRT channels are presented and discussed for the active region, quiet Sun and flare observations.
\end{abstract}

Key words. Sun: abundances - Sun: corona - Sun: X-rays, gamma rays

\section{Introduction}

In this paper we have used combined observations of a flare, an active region and a quiet Sun region by the Extreme-ultraviolet Imaging Spectrometer (EIS; Culhane et al. 2007) and the X-Ray Telescope (XRT; Golub et al. 2007) on board Hinode (Kosugi et al. 2007). The aim is to examine in detail the line and continuum contributions to the XRT channels and to assess the XRT temperature diagnostic potential. XRT is commonly used to constrain the hot component of the differential emission measure distribution (DEM). For example, Schmelz et al. (2009) and Reale et al. (2009) have used the Hinode XRT observations of active regions to obtain DEMs. Their analyses apparently provide evidence for the presence of plasma around $10 \mathrm{MK}$. Observations in multiple XRT bands can provide a measurement of temperatures in active regions and flares, however various factors can potentially affect the results. For example, the choice of elemental abundances and the range of temperatures over which the DEM inversion is performed. Completeness and accuracy of atomic data and the instrument calibration are also important issues. To address all these issues we perform a DEM inversion using simultaneous Hinode EIS observations, and compare the XRT count rates as predicted from the DEM modelling to the observed ones, by varying elemental abundances and the temperature range for the inversion.

We adopt the recent, significant revision of the Hinode EIS radiometric calibration (Del Zanna 2013b). The internal EIS calibration was obtained with the use of the line ratio technique, while the absolute calibration is mainly based on an Extreme Ultraviolet Normal Incidence Spectrograph (EUNIS) 2007 calibration rocket flight (Wang et al. 2011).
This work follows on from the study of a limb active region by O'Dwyer et al. (2011), hereafter referred to as Paper I. A similar work was carried out to assess the diagnostic potential of the Solar Dynamics Observatory (SDO) Atmospheric Imaging Assembly (AIA) broad-bands in Del Zanna et al. (2011). Due to its importance for coronal heating models involving nanoflares, we have specifically examined the coronal emission at temperatures above $5 \mathrm{MK}$.

Recently, Testa et al. (2011) performed a similar comparison between EIS and XRT observations. This paper is an extension and improvement on the Testa et al. (2011) manuscript in various respects. First, they only considered an active region core, while we have also considered a quiet Sun region and a solar flare (with much hotter emission). Second, their analysis used six XRT filters while eight are considered here. Third, their observations were performed on 2008 June 20 and the EIS ground calibration was adopted. The new EIS calibration increases the radiances of the EIS lines in the long-wavelength band by a significant $60 \%$. Prominent lines in this EIS band (e.g. from Fe XIV, Fe XV, Fe XVI) were used by Testa et al. to constrain the 2-3 MK emission measure. They found a significant discrepancy between the emission measure obtained from XRT, and that from EIS spectra, which was lower by almost a factor of two. These authors found that the disagreement was reduced if photospheric abundances were used. The new EIS calibration produces different results.

In Sect. 2 we provide the EIS and XRT observations and our data analysis techniques. Our results are presented in Sect. 3, with a discussion of these results in Sect. 4 and our conclusions in Sect. 5. 


\section{Observations and Data Analysis}

XRT is a grazing incidence X-ray imager equipped with a $2048 \times 2048$ CCD. The XRT has 1 arcsec pixels with a wide field of view of $34 \times 34$ arcmin. The XRT data were processed using standard XRT software available in SolarSoft (xrt_prep.pro). This includes the subtraction of a model dark frame, correction for vignetting, removal of high-frequency pattern noise, and normalisation by exposure time. Spacecraft jitter was removed using xrt_jitter.pro.

EIS is an off-axis spectrometer with a multi-toroidal grating which disperses the spectrum on two different detectors providing observations of emission lines from the solar corona and upper transition region (TR). The first detector covers the wavelength range 170-211 $\AA$ and the second covers 246-292 $\AA$.

The EIS data were first processed using the standard processing routine eis_prep.pro provided in SolarSoft software package (Freeland \& Handy 1998). This routine removes the dark current and CCD pedestal, as well as hot pixels and cosmic ray hits. We then applied the corrections to the Hinode EIS radiometric calibration as suggested by Del Zanna (2013b). A line fitting routine, written by one of us (GDZ) and using cfit (Haugan 1997), was applied to all of the lines at each pixel in the EIS raster. Sample EIS rasters are shown in Fig. 1. Instrumental effects such as the EIS slit-tilt and wavelength shifts due to spacecraft orbital variation have been compensated for. Once suitable regions simultaneously observed by EIS and XRT were identified, we applied the line fitting routine to the averaged EIS spectra for these regions.

Under the assumption that a plasma is optically thin and in ionisation equilibrium the observed intensity of a spectral emission line can be expressed as

$I_{\mathrm{ob}}=\int_{T_{\mathrm{e}}} A(z) G\left(T_{\mathrm{e}}, N_{\mathrm{e}}\right) \varphi\left(T_{\mathrm{e}}\right) \mathrm{d} T_{\mathrm{e}}$

where $A(z)$ is the abundance of the element $z$ producing the line, $T_{\mathrm{e}}$ is the electron temperature, and $N_{\mathrm{e}}$ is the electron number density. The contribution function, $G\left(T_{\mathrm{e}}, N_{\mathrm{e}}\right)$, contains the relevant atomic parameters for each line and can be obtained using equilibrium ionisation balance calculations. For this purpose we have used CHIANTI v7.1 (Dere et al. 1997; Landi et al. 2013) together with new atomic data for Fe VII (Witthoeft \& Badnell 2008; Del Zanna 2009). The new data for Fe XVII, benchmarked in (Del Zanna \& Ishikawa 2009), represent the most important recent update (since CHIANTI v7.0, see Landi et al. 2012) in terms of ions which contribute significantly to the XRT channels. The quantity $\varphi\left(T_{\mathrm{e}}\right)$ is known as the differential emission measure (DEM) which is defined as

$\varphi\left(T_{\mathrm{e}}\right)=N_{\mathrm{e}}{ }^{2} \frac{\mathrm{d} h}{\mathrm{~d} T_{\mathrm{e}}}$

where $h$ is the line-of-sight coordinate.

To evaluate the EIS DEM distributions, we used a MarkovChain Monte Carlo (MCMC) based reconstruction algorithm (Kashyap \& Drake 1998). The EIS lines, used to constrain the DEM curve, cover a broad temperature range. A list of the lines (and intensities) used for this purpose is provided in Table 1. The EIS observations constrain high temperatures with emission lines from Fe XVII, Ca XVII and Fe XXIII. The Fe XVII $275.55 \AA$ line (see Del Zanna \& Ishikawa 2009) was fitted by applying a three Gaussian fit to the lines in the window (Si VII 275.35 ̊, Fe XVII 275.55 ̊, Si VII 275.67 ̊). For a description of our approach to deblending the Ca XVII $192.82 \AA$ line see Paper I.
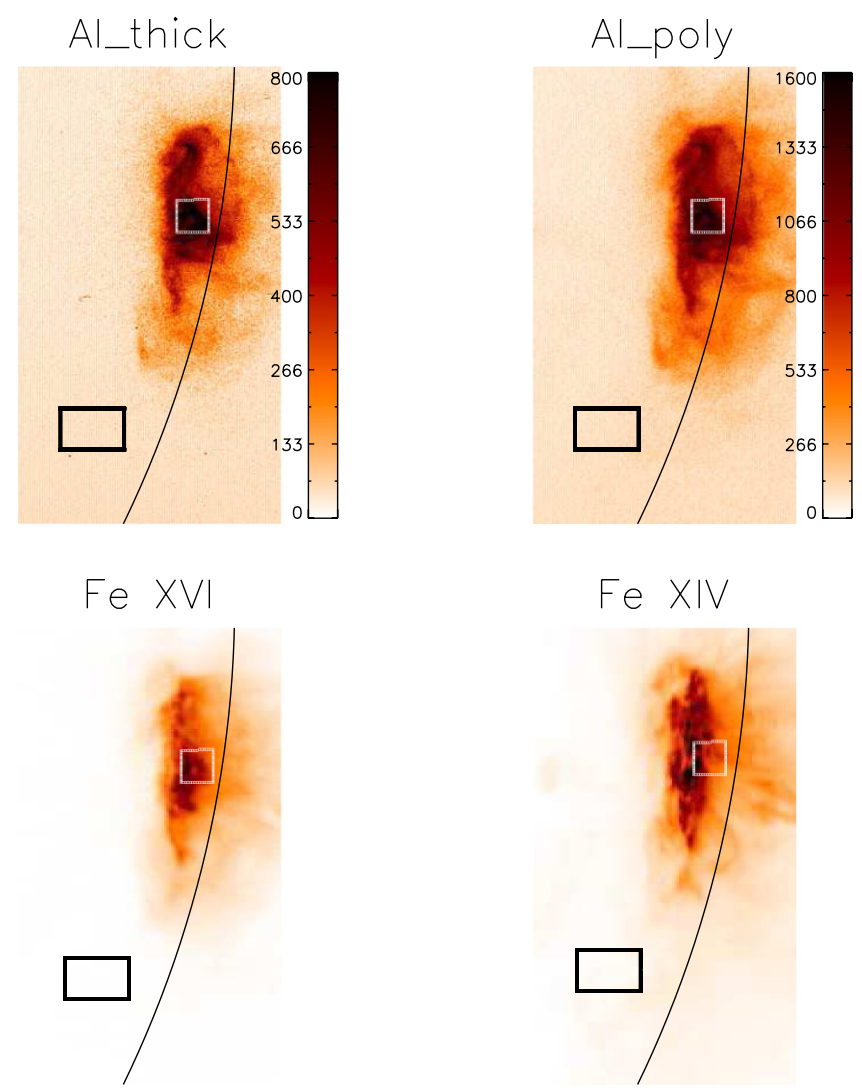

Fig. 1. Top: XRT images observed with the Al_thick and Al_poly filters. Intensities are in units of DN pix ${ }^{-1}$. Bottom: EIS intensity maps of the Fe XVI 265.0 $\AA$ and Fe XIV 264.79 ̊ lines. The locations of the active region area (white box) and quiet Sun area (black box) are indicated. The position of the limb is also indicated.

One of the aims of our study was to investigate the effect of varying elemental abundances on the XRT channels. Veck \& Parkinson (1981) found that low first ionisation potential (FIP) elements seem to have photospheric values, and that high-FIP elements seem to be depleted, using line-to-continuum X-ray measurements of solar flares observed by the Orbiting Solar Observatory (OSO)-8. Since then, a large body of literature on variations of coronal abundances (compared to their photospheric values) has been presented. Most observations suggest that the low-FIP elements such as iron are either enhanced (FIP effect) by a factor of four (e.g. Feldman et al. 1992) or are not enhanced (no FIP effect), relative to the high-FIP ones and taking the photospheric values as reference. The absolute (i.e. relative to hydrogen) coronal abundances are also still debated in the literature. In fact, it is still unclear whether the low-FIP elements are enhanced in the corona, or high FIP elements depleted, relative to their photospheric values, or if a 'hybrid' solution applies, as suggested by Schmelz et al. (2012).

For example, Lemen et al. (1986) using the Bent Crystal Spectrometer on SMM found that the coronal abundance of calcium relative to hydrogen was increased during the cooling phase of flares. Using spectra from the Solar and Heliospheric Observatory (SOHO) Solar Ultraviolet Measurements of Emitted Radiation (SUMER), Feldman et al. (1998) found that low-FIP elements were enriched by about a factor of four in the corona above a quiet equatorial region, but not above a polar coronal hole. In contrast, Fludra \& Schmelz (1995) applying a DEM and line-to-continuum analysis to observations with the Solar Maximum Mission (SMM) Bent Crystal Spectrometer 
B. O'Dwyer et al.: Response of Hinode XRT to quiet Sun, active region and flare plasma

Table 1. List of the emission lines and intensities used in the EIS DEM analysis for the active region, quiet Sun and flare cases.

\begin{tabular}{|c|c|c|c|c|c|c|c|c|}
\hline Ion & $\begin{array}{l}\lambda \\
\AA\end{array}$ & Transition & $\begin{array}{c}\log T_{\mathrm{m}}{ }^{a} \\
\mathrm{~K} \\
\end{array}$ & $\mathrm{AR}$ & QS & FL1 & FL2 & FL3 \\
\hline $\mathrm{O} \mathrm{V}$ & 192.91 & $2 \mathrm{~s} 2 \mathrm{p}^{3} \mathrm{P}_{2}-2 \mathrm{~s} 3 \mathrm{~d}^{3} \mathrm{D}_{3}$ & 5.4 & - & - & 440 & - & 7.7 \\
\hline $\mathrm{OV}$ & 248.46 & $2 \mathrm{~s} 2 \mathrm{p}{ }^{1} \mathrm{P}_{1}-2 \mathrm{~s} 3 \mathrm{~s}{ }^{1} \mathrm{~S}_{0}$ & 5.4 & 389 & 39 & - & - & - \\
\hline $\mathrm{Mg} \mathrm{V}$ & 276.58 & $2 \mathrm{~s}^{2} 2 \mathrm{p}^{4}{ }^{1} \mathrm{D}_{2}-2 \mathrm{~s} 2 \mathrm{p}^{5}{ }^{1} \mathrm{P}_{1}$ & 5.4 & 104 & 5 & - & - & - \\
\hline $\operatorname{Mg~VI~}(2)^{b}$ & 270.39 & $\begin{array}{l}2 \mathrm{~s}^{2} 2 \mathrm{p}^{3}{ }^{2} \mathrm{D}_{5 / 2}-2 \mathrm{~s} 2 \mathrm{p}^{4}{ }^{2} \mathrm{P}_{3 / 2} \\
2 \mathrm{~s}^{2} 2 \mathrm{p}^{3}{ }^{2} \mathrm{D}_{3 / 2}-2 \mathrm{~s} 2 \mathrm{p}^{4}{ }^{2} \mathrm{P}_{3 / 2}\end{array}$ & 5.6 & 382 & 22 & - & - & - \\
\hline Fe VIII & 185.21 & $3 s^{2} 3 p^{6} 3 d^{2} D_{5 / 2}-3 s^{2} 3 p^{5} 3 d^{2}{ }^{2} F_{7 / 2}$ & 5.7 & - & - & 1290 & 1490 & 899 \\
\hline Mg VII & 278.4 & $2 s^{2} 2 p^{2}{ }^{3} P_{2}-2 s 2 p^{3}{ }^{3} S_{1}$ & 5.8 & 988 & 108 & - & - & - \\
\hline Si VII & 275.35 & $2 s^{2} 2 p^{4}{ }^{3} P_{2}-2 s 2 p^{5}{ }^{3} P_{2}$ & 5.8 & 739 & 92 & - & - & 295 \\
\hline Fe X & 184.54 & $3 s^{2} 3 p^{5}{ }^{2} P_{3 / 2}-3 s^{2} 3 p^{4} 3 d^{2} S_{1 / 2}$ & 6.0 & 2240 & 365 & 1150 & 873 & 844 \\
\hline $\mathrm{Fe} \mathrm{XI}$ & 180.43 & $3 s^{2} 3 p^{4}{ }^{3} P_{2}-3 s^{2} 3 p^{3} 3 d^{3} D_{3}$ & 6.1 & 6390 & 1210 & - & - & - \\
\hline $\mathrm{Fe} X \mathrm{XI}$ & 188.22 & $3 s^{2} 3 p^{4}{ }^{3} \mathrm{P}_{2}-3 s^{2} 3 p^{3} 3 d^{3} \mathrm{P}_{2}$ & 6.1 & 3730 & 430 & 2120 & 2350 & 1460 \\
\hline Fe XI & 188.3 & $3 s^{2} 3 p^{4}{ }^{3} P_{2}-3 s^{2} 3 p^{3} 3 d^{1} P_{1}$ & 6.1 & - & - & 1080 & 1040 & 964 \\
\hline Fe XII & 192.39 & $3 s^{2} 3 p^{3}{ }^{4} S_{3 / 2}-3 s^{2} 3 p^{2} 3 d^{4} P_{1 / 2}$ & 6.2 & 2190 & 207 & - & - & - \\
\hline Fe XII & 195.12 & $3 s^{2} 3 p^{3}{ }^{4} S_{3 / 2}-3 s^{2} 3 p^{2} 3 d^{4} P_{5 / 2}$ & 6.2 & - & - & 2730 & 2310 & 2150 \\
\hline Si X & 256.4 & $2 s^{2} 2 p^{2} P_{1 / 2}-2 s 2 p^{2}{ }^{2} P_{1 / 2}$ & 6.1 & - & - & 1820 & 1220 & 2380 \\
\hline Si X & 261.06 & $2 \mathrm{~s}^{2} 2 \mathrm{p}^{2} \mathrm{P}_{3 / 2}-2 \mathrm{~s} 2 \mathrm{p}^{2}{ }^{2} \mathrm{P}_{1 / 2}$ & 6.1 & 1080 & 104 & - & - & - \\
\hline Fe XIII & 202.04 & $3 s^{2} 3 p^{2}{ }^{3} P_{0}-3 s^{2} 3 p 3 d^{3} P_{1}$ & 6.2 & 3900 & 457 & 1530 & 1190 & 1120 \\
\hline Fe XIV & 264.79 & $3 s^{2} 3 p^{2} \mathrm{P}_{3 / 2}-3 s 3 p^{2}{ }^{2} \mathrm{P}_{3 / 2}$ & 6.3 & - & - & 830 & 4450 & 6210 \\
\hline Fe XIV & 274.2 & $3 s^{2} 3 p^{2} P_{1 / 2}-3 s 3 p^{2}{ }^{2} S_{1 / 2}$ & 6.3 & 5280 & 186 & 7030 & 2670 & 3990 \\
\hline Fe XV & 284.16 & $3 \mathrm{~s}^{2}{ }^{1} \mathrm{~S}_{0}-3 \mathrm{~s} 3 \mathrm{p}{ }^{1} \mathrm{P}_{1}$ & 6.3 & 25100 & 747 & 9180 & 26400 & 45300 \\
\hline Fe XVI & 262.98 & $3 p^{2} P_{3 / 2}-3 d^{2} D_{5 / 2}$ & 6.4 & 7190 & 23 & 1640 & 4890 & 12200 \\
\hline Fe XVI & 265.0 & $3 p^{2} \mathrm{P}_{3 / 2}-3 \mathrm{~d}^{2} \mathrm{D}_{3 / 2}$ & 6.4 & - & - & 117 & 642 & 1090 \\
\hline $\mathrm{Ca} X V$ & 200.98 & $2 \mathrm{~s}^{2} 2 \mathrm{p}^{2}{ }^{3} \mathrm{P}_{0}-2 \mathrm{~s} 2 \mathrm{p}^{3}{ }^{3} \mathrm{D}_{1}$ & 6.6 & 774 & 3 & - & - & - \\
\hline Fe XVII & 275.55 & $2 s^{2} 2 p^{5} 3 p{ }^{1} P_{1}-2 s^{2} 2 p^{5} 3 d^{1} D_{2}$ & 6.6 & 46 & 0.5 & - & - & - \\
\hline Fe XVII & 284.39 & $2 s^{2} 2 p^{5} 3 p^{3} D_{3}-2 s^{2} 2 p^{5} 3 d^{3} P_{2}$ & 6.6 & - & - & 110 & 646 & 410 \\
\hline Ca XVII & 192.82 & $2 \mathrm{~s}^{2}{ }^{1} \mathrm{~S}_{0}-2 \mathrm{~s} 2 \mathrm{p}{ }^{1} \mathrm{P}_{1}$ & 6.7 & 823 & - & 4630 & 1780 & 976 \\
\hline Fe XXIII & 263.77 & $2 \mathrm{~s}^{2}{ }^{1} \mathrm{~S}_{0}-2 \mathrm{~s} 2 \mathrm{p}{ }^{3} \mathrm{P}_{1}$ & 7.15 & 2.5 & - & 690 & 1730 & 0.3 \\
\hline Fe XXIV & 192.03 & $1 s^{2} 2 s^{2} S_{1 / 2}-1 s^{2} 2 p^{2} P_{3 / 2}$ & 7.25 & - & - & 857 & 658 & 81.8 \\
\hline Fe XXIV & 255.11 & $1 s^{2} 2 s^{2} S_{1 / 2}-1 s^{2} 2 p^{2} P_{1 / 2}$ & 7.25 & - & - & 2060 & 4870 & 82.0 \\
\hline
\end{tabular}

Notes. ${ }^{(a)} T_{\mathrm{m}}$ corresponds to the temperature of maximum abundance. ${ }^{(b)}$ Denotes a self blend. The numbers in the columns are the EIS calibrated intensities for the active region (AR), quiet Sun (QS) and three flare (FL1, FL2, FL3) cases discussed within the text.

(BCS) found high-FIP elements depleted compared with their photospheric values. In terms of quiescent active region cores, a recent analysis (Del Zanna 2013a) based on Hinode EIS observations suggests a FIP enhancement of about a factor of 3 , and that low-FIP elements such as iron have to be enhanced (compared to the photospheric values) by at least a factor of three, in agreement with direct line-to-continuum measurements based on ground-based eclipse observations (Mason 1975).

In our analysis we have used four abundance datasets to assess how varying elemental abundances affects the analysis of the XRT data. The chosen datasets span the main range of abundance values found in the literature, both in terms of FIP effect (from zero to four) and absolute value. These are the photospheric abundances of Grevesse \& Sauval (1998; photospheric 1) and Asplund et al. (2009; photospheric 2), the coronal abundances of Feldman et al. (1992; coronal 1) and the photospheric abundances of Asplund et al. (2009) with the oxygen and neon abundances reduced by a factor of three (coronal 2). The coronal 1 abundances increase the abundance values of the low FIP elements compared to their photospheric values. The coronal 2 abundances reduce the abundance values of the two main high FIP elements compared to their photospheric values (as we shall discuss below, oxygen and neon are the main two high
FIP elements contributing to the XRT bands). The primary difference between the photospheric abundances of Asplund et al. (2009) and those of Grevesse \& Sauval (1998) is a reduction in the abundance values of some of the high-FIP elements, including oxygen and neon.

For each of the active region, quiet Sun and flare cases the inversion of the Hinode EIS data and the forward-modelling of the Hinode XRT channels were performed with the above four different abundance datasets. We note that the dominant Hinode EIS lines for DEM analysis are from iron (cf. Table 1), and that, as we shall see later, neon and oxygen are the dominant high-FIP elements contributing to the XRT bands.

Electron density values obtained using the Fe XIII $203.83 \AA$ to $202.04 \AA$ ratio were used to calculate the contribution functions for the lines used to constrain the EIS DEM curves (which have very little density sensitivity anyway). For the active region, quiet Sun and flare cases the measured densities were $3.6 \times 10^{8}$, $6.0 \times 10^{9}, 8 \times 10^{10} \mathrm{~cm}^{-3}$ respectively.

We used the ionisation equilibrium calculations of Dere et al. (2009). Once the DEMs were obtained, we then calculated CHIANTI synthetic spectra using the same set of parameters (abundances and densities). These synthetic spectra were 
A\&A 561, A20 (2014)

Table 2. Observed and predicted XRT count rates for the active region area shown in Fig. 1.

\begin{tabular}{lccccc}
\hline \hline & \multirow{2}{*}{ Observed } & \multicolumn{4}{c}{ Predicted } \\
& & Photospheric 1 & Photospheric 2 & Coronal 1 & Coronal 2 \\
\hline Al_poly & 12100 & $24400(+102 \%)$ & $21600(+79 \%)$ & $14000(+16 \%)$ & $16200(+34 \%)$ \\
Al_thick & 22.2 & $30.6(+38 \%)$ & $30.3(+36 \%)$ & $17.5(-21 \%)$ & $24.0(+8 \%)$ \\
Al_Ti & 4160 & $7770(+87 \%)$ & $7080(+70 \%)$ & $4560(+10 \%)$ & $5360(+29 \%)$ \\
Be_thin & 2480 & $5050(+104 \%)$ & $4660(+88 \%)$ & $2940(+19 \%)$ & $3500(+41 \%)$ \\
Be_med & 540 & $1020(+89 \%)$ & $970(+80 \%)$ & $572(+6 \%)$ & $739(+37 \%)$ \\
Be_thick & 0.715 & $1.07(+50 \%)$ & $1.17(+64 \%)$ & $0.629(-12 \%)$ & $0.923(+29 \%)$ \\
C_poly & 8410 & $16100(+91 \%)$ & $14500(+72 \%)$ & $9540(+13 \%)$ & $11000(+31 \%)$ \\
Ti_poly & 5667 & $10500(+85 \%)$ & $9490(+67 \%)$ & $6090(+7 \%)$ & $7290(+29 \%)$ \\
\hline
\end{tabular}

Notes. The predicted XRT count rates are determined from the corresponding EIS DEM curve. All values are in units of DN $\mathrm{s}^{-1}$ pix ${ }^{-1}$. Photospheric 1 and 2 correspond to the photospheric abundances of Grevesse et al. (1998) and Asplund et al. (2009) respectively. Coronal 1 corresponds to the coronal abundances of Feldman et al. (1992). Coronal 2 corresponds to the photospheric abundances of Asplund et al. (2009) with the oxygen and neon abundances reduced by a factor of three. The percentage difference between the observed and predicted count rates are included in parentheses.

Table 3. Same as Table 2 except the EIS DEM curves are set to zero above $\log T[\mathrm{~K}]=6.8$.

\begin{tabular}{lccccc}
\hline \hline & \multirow{2}{*}{ Observed } & \multicolumn{4}{c}{ Predicted } \\
& & Photospheric 1 & Photospheric 2 & Coronal 1 & Coronal 2 \\
\hline Al_poly & 12100 & $24200(+100 \%)$ & $19700(+63 \%)$ & $13600(+12 \%)$ & $15300(+26 \%)$ \\
Al_thick & 22.2 & $30.1(+36 \%)$ & $25.6(+15 \%)$ & $16.7(-25 \%)$ & $21.3(-4 \%)$ \\
Al_Ti & 4160 & $7680(+85 \%)$ & $6270(+51 \%)$ & $4390(+6 \%)$ & $4980(+20 \%)$ \\
Be_thin & 2480 & $4980(+101 \%)$ & $4060(+64 \%)$ & $2820(+14 \%)$ & $3200(+29 \%)$ \\
Be_med & 540 & $1000(+85 \%)$ & $824(+53 \%)$ & $545(+1 \%)$ & $661(+22 \%)$ \\
Be_thick & 0.715 & $1.04(+45 \%)$ & $0.860(+20 \%)$ & $0.579(-19 \%)$ & $0.722(+1 \%)$ \\
C_poly & 8410 & $16000(+90 \%)$ & $13100(+56 \%)$ & $9230(+10 \%)$ & $10300(+22 \%)$ \\
Ti_poly & 5667 & $10400(+84 \%)$ & $8500(+50 \%)$ & $5890(+4 \%)$ & $6820(+20 \%)$ \\
\hline
\end{tabular}

then convolved with the effective area of each XRT channel, to produce XRT count rates.

DEM curves were also obtained using the observed XRT count rates and the response functions calculated with CHIANTI v.7.1. These DEMs are then compared to those obtained from the EIS lines. Updated XRT filter calibrations were used (Narukage et al. 2011). Also in this case we used the Markov-chain Monte Carlo (MCMC) based reconstruction algorithm (Kashyap \& Drake 1998) and the four abundance datasets.

\subsection{Active region and quiet Sun}

An observation was made on 17 December, 2007 using EIS. The target of opportunity was an active region close to the solar limb, with the core still on the disk. The observation sequence we designed (CAM_AR_LIMB) used the $2^{\prime \prime}$ slit with an exposure time of $45 \mathrm{~s}$. The EIS raster used in this analysis started at 10:42:20 UT. The raster was $360^{\prime \prime}$ wide, used a slit length of $512^{\prime \prime}$ and lasted for $2.5 \mathrm{~h}$. We designed a Hinode Observing Program with both EIS and XRT. We note that we searched the Hinode database and found that XRT observations with multiple filters and simultaneous EIS observations with suitable lines are rare. Due to the large number of XRT filters involved we found this observation to be the most suitable for the present study.

Figure 1 shows the location of an area within the active region and a separate area of quiet Sun over which spectra were averaged. Large areas were chosen in order to increase the number of counts and to reduce the effect of any possible misalignments between the EIS and XRT data. The active region and quiet Sun areas correspond to 812 and 2013 arcsec squared respectively.
Over the duration of the EIS raster, observations of the active region were also made using XRT. XRT images of the active region were obtained with the Al_poly, C_poly, Be_thin, Be_medium, Ti_poly, Al_thick and Be_thick filters as well as the filter combination Al_poly/Ti_poly. This represents all of the XRT filters with the exception of Al_mesh and Al_med filters. Sample XRT images are shown in Fig. 1. Short and long exposure pairs of observations were taken for Al_poly, C_poly, Be_thin, Ti_poly and Al_poly/Ti_poly. The long exposure images for these filters were affected by saturation. Long and short exposures were co-aligned and combined in order to account for the presence of saturated pixels. The combination of short and long exposures involved replacing the values of the observed flux for the saturated pixels in the long exposure images by the corresponding values in the short exposure images. This modification involved all four filters (Al_poly, C_poly, Be_thin, Ti_poly), as well as the filter combination Al_poly/Ti_poly, for which short/long pairs of observations were taken. Images taken with the other three filters (Be_thick, Al_thick, Be_med) were not affected by saturation. The intensities of these combined XRT images were summed over the active region (see Tables 2 and 3) and quiet Sun areas (see Tables 4 and 5).

\subsection{Flare}

A flare observation was analysed in order to further examine the response of XRT to high temperature plasma. In terms of examining the relative intensity calibration of EIS and XRT this type of observation has the advantange of a large number of $\mathrm{DN} \mathrm{s}^{-1}$. In addition, the higher temperature emission 
B. O'Dwyer et al.: Response of Hinode XRT to quiet Sun, active region and flare plasma

Table 4. Same as Table 2 for the quiet Sun area shown in Fig. 1.

\begin{tabular}{lccccc}
\hline \hline & Observed & \multicolumn{4}{c}{ Predicted } \\
& & Photospheric 1 & Photospheric 2 & Coronal 1 & Coronal 2 \\
\hline Al_poly & 31.7 & $104(+228 \%)$ & $75.4(+138 \%)$ & $31.1(-2 \%)$ & $38.0(+20 \%)$ \\
Al_thick & 0.0609 & $0.113(+86 \%)$ & $0.0949(+56 \%)$ & $0.024(-61 \%)$ & $0.0505(-17 \%)$ \\
Al_Ti & 7.95 & $29.7(+274 \%)$ & $21.8(+174 \%)$ & $8.27(+4 \%)$ & $11.1(+40 \%)$ \\
Be_thin & 3.23 & $17.6(+445 \%)$ & $12.9(+299 \%)$ & $4.33(+34 \%)$ & $6.01(+86 \%)$ \\
Be_med & 0.907 & $3.70(+308 \%)$ & $2.94(+224 \%)$ & $0.797(-12 \%)$ & $1.48(+63 \%)$ \\
Be_thick & 0.0227 & $0.00537(-76 \%)$ & $0.00567(-75 \%)$ & $0.000768(-97 \%)$ & $0.00352(-84 \%)$ \\
C_poly & 28.0 & $71.2(+154 \%)$ & $52.5(+88 \%)$ & $23.6(-16 \%)$ & $29.6(+6 \%)$ \\
Ti_poly & 18.6 & $47.5(+155 \%)$ & $35.6(+91 \%)$ & $15.1(-19 \%)$ & $21.4(+15 \%)$ \\
\hline
\end{tabular}

Table 5. Same as Table 4 for the quiet Sun area shown in Fig. 1, except that the EIS DEM curves are set to zero above $\log T[\mathrm{~K}]=6.5$.

\begin{tabular}{lccccc}
\hline \hline & \multirow{2}{*}{ Observed } & \multicolumn{2}{c}{ Predicted } \\
& & Photospheric 1 & Photospheric 2 & Coronal 1 & Coronal 2 \\
\hline Al_poly & 31.7 & $51.2(+62 \%)$ & $41.5(+31 \%)$ & $21.0(-34 \%)$ & $26.9(-15 \%)$ \\
Al_thick & 0.0609 & $0.0163(-73 \%)$ & $0.0153(-75 \%)$ & $0.00976(-84 \%)$ & $0.0131(-78 \%)$ \\
Al_Ti & 7.95 & $10.5(+32 \%)$ & $8.80(+11 \%)$ & $4.78(-40 \%)$ & $6.42(-19 \%)$ \\
Be_thin & 3.23 & $4.08(+26 \%)$ & $3.50(+8 \%)$ & $1.99(-38 \%)$ & $2.42(-25 \%)$ \\
Be_med & 0.907 & $0.606(-33 \%)$ & $0.542(-40 \%)$ & $0.320(-65 \%)$ & $0.416(-54 \%)$ \\
Be_thick & 0.0227 & $0.000246(-99 \%)$ & $0.000230(-99 \%)$ & $0.000152(-99 \%)$ & $0.000211(-99 \%)$ \\
C_poly & 28.0 & $34.4(+23 \%)$ & $28.5(+2 \%)$ & $16.6(-41 \%)$ & $21.4(-24 \%)$ \\
Ti_poly & 18.6 & $23.1(+24 \%)$ & $19.3(+4 \%)$ & $10.6(-43 \%)$ & $15.6(-16 \%)$ \\
\hline
\end{tabular}

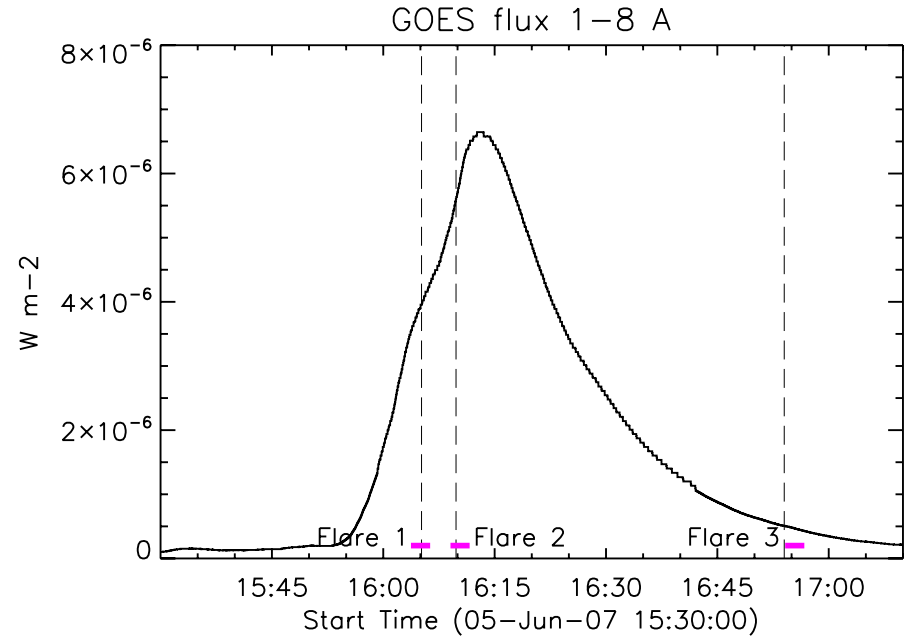

Fig. 2. GOES X-ray light curve of the GOES C6.6 class flare on 5 June, 2007. The times of the EIS rasters (Flare 1, Flare 2, Flare 3) are shown by horizontal bars (magenta). The start times of the Be_thick, Ti_poly and Al_thick observations are shown by dashed vertical lines.

is well constrained with EIS. Observations of a Geostationary Operational Environmental Satellite (GOES) C6.6 class flare were made on 5 June, 2007, using EIS. The observation sequence, HH_AR+FLR_RAS_H01, used the $1^{\prime \prime}$ slit with an exposure time of $5 \mathrm{~s}$. The sequence used a sparse raster with $10^{\prime \prime}$ separations between exposure positions. The raster was $240^{\prime \prime}$ wide, with a slit length of $240^{\prime \prime}$, and lasted $156 \mathrm{~s}$. Flare lines observed by the sequence include Fe XVII $254.885 \AA(\log T[\mathrm{~K}]=$ 6.6), Ca XVII 192.853 $\mathrm{A}(\log T[\mathrm{~K}]=6.75), \mathrm{Fe}$ XXIII $263.765 \AA$ $(\log T[\mathrm{~K}]=7.15), \mathrm{Fe} X X I V 192.028 \AA(\log T[\mathrm{~K}]=7.25)$ and Fe XXIV $255.113 \AA(\log T[\mathrm{~K}]=7.25)$. The GOES X-ray light curve of the flare is shown in Fig. 2. The flare begins at 15:50 UT,

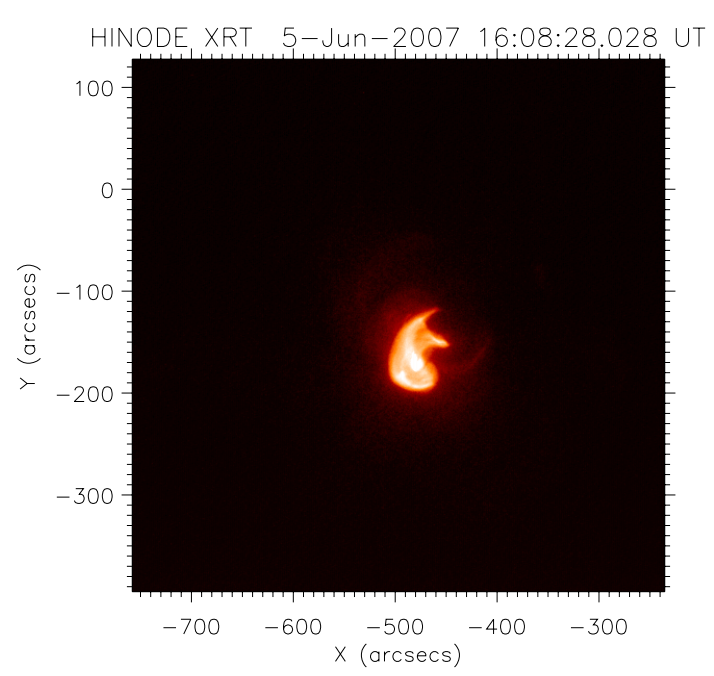

Fig. 3. XRT image observed with the Ti_poly filter at 16:08:28 UT.

peaks at 16:13 UT, and ends at 17:00 UT. Three EIS rasters were analysed beginning at 16:03:44 (Flare 1), 16:09:03 (Flare 2) and 16:54:08 (Flare 3). XRT performed observations with only a few filters and varying cadence during the flare, so it was not possible to derive a DEM curve from the XRT data. Figure 3 shows an XRT Ti_poly image of the flaring active region at 16:08:28 UT. For the EIS vs. XRT comparison, we selected a Be_thick image taken at 16:05, a Ti_poly image taken at 16:09, and an Al_thick image taken at 16:54 UT.

A region of the flare close to the core of the active region was selected for detailed study. The spectra from the EIS sparse raster over this region were averaged. A careful alignment was made between the Hinode EIS and XRT observations. The intensities of the corresponding XRT images were summed over the same area. Observed XRT intensity values are given in Table 6. 

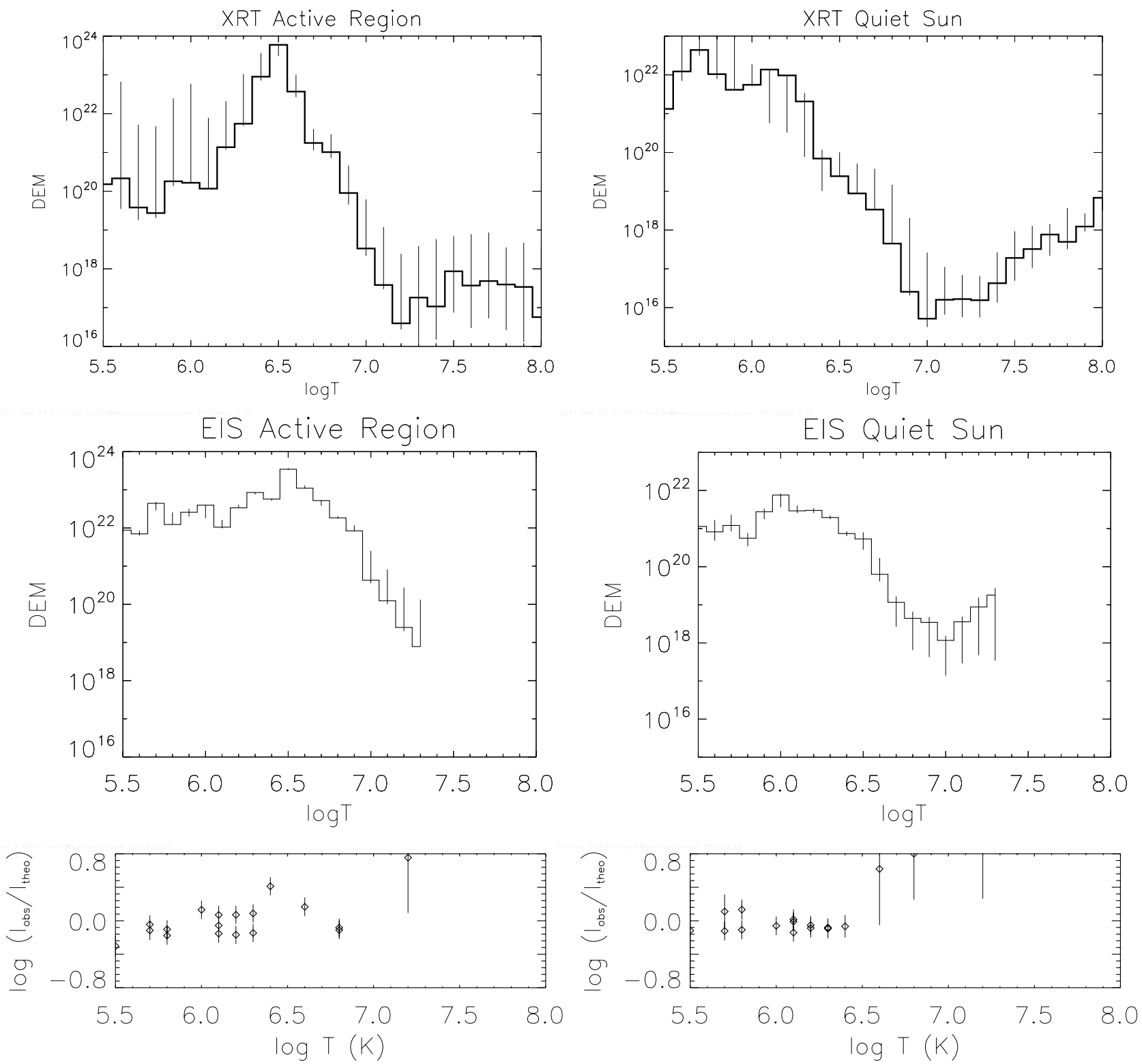

Fig. 4. Top row: XRT DEM curves for the active region (left) and quiet Sun (right) areas shown in Fig. 1. Middle row: EIS DEM curves for the active region (left) and quiet Sun (right) areas. Bottom row: plots of the ratio of the observed intensity to the theoretical intensity for each spectral line used to constrain the corresponding EIS DEM curve. The error bars on the ratio correspond to a combination of the one-sigma uncertainties on the line fitting and an uncertainty of $22 \%$ on the observed intensity based on the pre-flight calibration of EIS (Lang et al. 2006).

Table 6. Same as Table 2 for the flare observations.

\begin{tabular}{lccccc}
\hline \hline & Observed & \multicolumn{3}{c}{ Predicted } \\
& & Photospheric 1 & Photospheric 2 & Coronal 1 & Coronal 2 \\
\hline Be_thick (Flare 1) & 4.75 & $2.80(-41 \%)$ & $3.36(-29 \%)$ & $2.30(-52 \%)$ & $5.91(+24 \%)$ \\
Ti_poly (Flare 2) & 20781 & $6493(-69 \%)$ & $23184(+12 \%)$ & $16973(-18 \%)$ & $5732(-72 \%)$ \\
Al_thick (Flare 3) & 53.5 & $99.8(+87 \%)$ & $96.9(+81 \%)$ & $64.0(+20 \%)$ & $69.6(+30 \%)$ \\
\hline
\end{tabular}

\section{Results}

\subsection{DEM curves}

\subsubsection{Active region and quiet Sun}

We were unable to definitely identify the Fe XXIII $263.765 \AA$ line (temperature of formation $\log T \sim 7.15$ ) in the active region spectrum. As a result the EIS active region DEM curve is not well constrained above $\log T \sim 6.85$, with the only constraint being an upper limit on the Fe XXIII $263.765 \AA$ line. For the quiet Sun case upper limits were obtained for the Fe XVII $275.55 \AA$ A , Ca XVII 192.82 A and Fe XXIII 263.765 Å lines.

Figure 4 shows the active region EIS DEM curve created using the photospheric 2 abundances. The curve peaks at $\log T \sim$ 6.5 , then falls off rapidly above $\log T \sim 6.7$. The quiet Sun EIS DEM curve created using the coronal 2 abundances is also displayed in Fig. 4. The curve has a broad peak at lower 
temperatures, then falls off rapidly above $\log T \sim 6.3$. With the exception of O V $248.46 \AA$ all of the lines used to constrain the EIS DEM curves were emitted by low-FIP elements (see Table 1). It is worth noting that for both the active region and quiet Sun cases the observed intensities of the O V $248.46 \AA$ and $\mathrm{Mg} \mathrm{V} 276.58 \AA$ lines $(\log T[\mathrm{~K}]=6.4)$ were better reproduced using the photospheric 2 abundance values of Asplund et al. (2009). The active region XRT DEM curve created using the photospheric 2 abundances is also displayed in Fig. 4. The curve peaks at $\log T \sim 6.5$. The quiet Sun XRT DEM curve created using the coronal 2 abundances is also displayed in Fig. 4. The curve has a broad peak at lower temperatures, then falls off rapidly above $\log T \sim 6.2$. Within the uncertainties there is relatively good agreement between the EIS and XRT DEM curves for both the active region and quiet Sun cases.

Of the EIS DEMs obtained for the active region and quiet Sun cases in each case those obtained using photospheric 1, photospheric 2 and coronal 2 abundances are very similar. This is due to the fact that the abundance values of the low FIP elements are similar in each of these abundance datasets. The lines we have used to constrain the EIS DEM curves are primarily from low FIP elements. The EIS DEM curves obtained using the coronal 1 abundances are qualitatively similar to the EIS DEM curves obtained using the other abundance datasets except that with the coronal 1 abundances the DEM curves are shifted down by a factor of four. This is due to the fact that the abundance values of the low FIP elements are increased by almost a factor of four for the coronal 1 abundances compared with photospheric values.

\subsubsection{Flare}

A line fitting routine was applied to the averaged EIS spectra for each of the flare cases corresponding to the EIS rasters beginning at 16:03:44 (Flare 1), 16:09:03 (Flare 2) and 16:54:08 (Flare 3). Corresponding DEM curves were created for the four abundance datasets. Our DEM modelling assumes that the plasma is in ionisation equilibrium. This might not be an appropriate assumption for a flaring plasma. The electron density measured for this flare is around $8 \times 10^{10} \mathrm{~cm}^{-3}$. Using e.g. Fig. 2 of Bradshaw $\&$ Raymond (2013) we estimate that the assumption of ionisation equilibrium is appropriate for plasma heating processes on timescales of 1-10 s. Although it is possible that the flare does not reach ionisation equilibrium during the impulsive phase, we consider this to be unlikely in this case, since the rise phase takes $10 \mathrm{~min}$.

The EIS DEM curves for flare 1 and flare 2 (Fig. 5), during the rise and peak of the soft X-ray emission for the flare (Fig. 2), show significant emission around $\log T[\mathrm{~K}]=7.0$. The EIS DEM for flare 3 (Fig. 5), during the decay phase (Fig. 2), shows a peak temperature around $\log T[\mathrm{~K}]=6.8$.

\subsection{Synthetic spectra and predicted XRT count rates}

\subsubsection{Active region and quiet Sun}

Synthetic spectra have been calculated from the corresponding EIS DEMs. For both the active region and quiet Sun cases the synthetic spectrum for each XRT filter was integrated in order to predict the total count rate. The predicted count rates are included in Tables 2 and 4 respectively. Our EIS DEM curves are not well constrained above $\log T \sim 6.8 \mathrm{~K}$, since the only constraint is an upper limit on the line intensity of
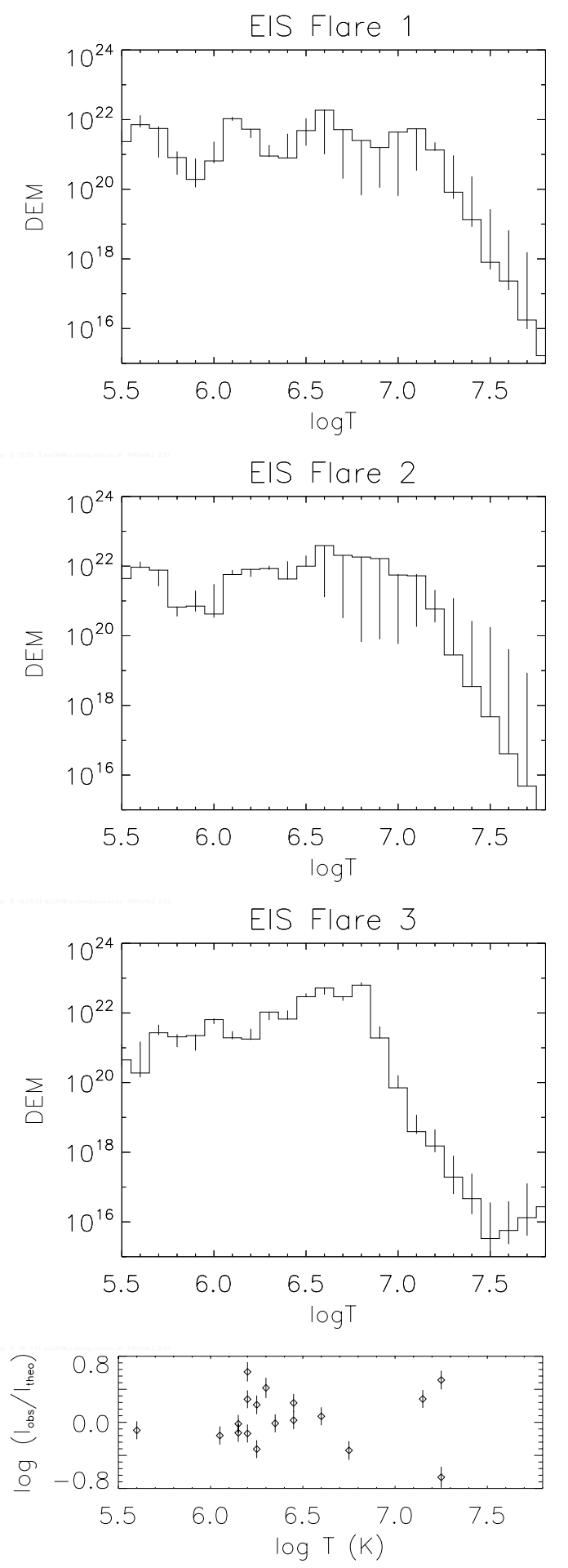

Fig. 5. EIS DEM curve for the three flare cases. Also included is a plot of the ratio of the observed intensity to the theoretical intensity for each spectral line used to constrain the EIS Flare 2 DEM curve.

Fe XXIII $263.77 \AA$. In order to investigate the effect of the poorly constrained higher temperature component of the EIS DEM curves on the predicted XRT count rates we have also considered the effect of imposing that the active region EIS DEM curve fall to zero above $\log T=6.8 \mathrm{~K}$. The predicted XRT count rates obtained in this way are included in Table 3 . The reduction in the predicted count rates is almost $15 \%$ for the Be_thick channel, but less than $10 \%$ for the other channels. We have repeated this analysis for the quiet Sun case setting the EIS DEM curve to zero 
Table 7. Contribution of lines and continuum to each of the XRT filters.

\begin{tabular}{|c|c|c|c|c|c|c|}
\hline & \multirow[t]{2}{*}{ Ion } & \multirow{2}{*}{$\begin{array}{l}\lambda \\
\AA\end{array}$} & \multirow{2}{*}{$\begin{array}{c}\log T_{\max } \\
\mathrm{K}\end{array}$} & \multicolumn{3}{|c|}{$\begin{array}{l}\text { Percentage of } \\
\text { total emission }\end{array}$} \\
\hline & & & & $\begin{array}{l}\text { Active } \\
\text { region }\end{array}$ & $\begin{array}{l}\text { Quiet } \\
\text { Sun }\end{array}$ & Flare \\
\hline \multirow[t]{7}{*}{ C_poly } & Fe XVII & 17.10 & 6.6 & 5 & - & 4 \\
\hline & Fe XVII & 17.05 & 6.6 & 5 & - & 4 \\
\hline & Fe XVII & 16.78 & 6.6 & 4 & - & 3 \\
\hline & Fe XVII & 15.01 & 6.6 & 10 & 5 & 9 \\
\hline & Ne IX & 13.45 & 6.2 & 4 & - & - \\
\hline & O VIII & 18.97 & 6.4 & 5 & - & - \\
\hline & Cont. & & & 18 & 22 & 7 \\
\hline \multirow[t]{9}{*}{ Al_poly } & Fe XVII & 17.10 & 6.6 & 5 & 3 & 5 \\
\hline & Fe XVII & 17.05 & 6.6 & 6 & 4 & 5 \\
\hline & Fe XVII & 16.78 & 6.6 & 5 & 3 & 4 \\
\hline & Fe XVII & 15.26 & 6.6 & - & - & 3 \\
\hline & Fe XVII & 15.01 & 6.6 & 9 & 5 & 9 \\
\hline & $\mathrm{Ne} I X$ & 13.45 & 6.2 & 4 & - & - \\
\hline & O VIII & 18.97 & 6.4 & 6 & 4 & - \\
\hline & O VII & 21.60 & 6.0 & - & 5 & - \\
\hline & Cont. & & & 16 & 23 & 7 \\
\hline \multirow[t]{6}{*}{ Ti_poly } & Fe XVII & 17.10 & 6.6 & 4 & - & 3 \\
\hline & Fe XVII & 17.05 & 6.6 & 4 & - & 3 \\
\hline & Fe XVII & 16.78 & 6.6 & 3 & - & 3 \\
\hline & Fe XVII & 15.01 & 6.6 & 9 & 4 & 8 \\
\hline & Ne IX & 13.45 & 6.2 & 4 & - & - \\
\hline & Cont. & & & 20 & 23 & 9 \\
\hline \multirow[t]{4}{*}{ Be_thin } & Fe XVII & 15.01 & 6.6 & 8 & 6 & 6 \\
\hline & Mg XI & 9.17 & 6.5 & 4 & - & 4 \\
\hline & Ne IX & 13.45 & 6.2 & 5 & - & - \\
\hline & Cont. & & & 24 & 35 & 10 \\
\hline Al_poly - & Fe XVII & 17.10 & 6.6 & 3 & - & - \\
\hline \multirow[t]{6}{*}{ Ti_poly } & Fe XVII & 17.05 & 6.6 & 4 & - & 3 \\
\hline & Fe XVII & 16.78 & 6.6 & 3 & - & - \\
\hline & Fe XVII & 15.01 & 6.6 & 9 & 6 & 7 \\
\hline & Mg XI & 9.17 & 6.5 & - & - & 3 \\
\hline & $\mathrm{Ne} I X$ & 13.45 & 6.2 & 5 & - & - \\
\hline & Cont. & & & 20 & 28 & 9 \\
\hline \multirow[t]{5}{*}{ Be_med } & Mg XII & 8.42 & 6.9 & - & - & 5 \\
\hline & $\operatorname{Mg}$ XI & 9.31 & 6.5 & 5 & - & 4 \\
\hline & Mg XI & 9.17 & 6.5 & 9 & 5 & 8 \\
\hline & Si XIII & 6.65 & 6.7 & - & - & 4 \\
\hline & Cont. & & & 37 & 46 & 17 \\
\hline \multirow[t]{6}{*}{ Al_thick } & Mg XII & 8.43 & 6.9 & - & 3 & 8 \\
\hline & Mg XII & 8.42 & 6.9 & 5 & 7 & 16 \\
\hline & Mg XI & 9.31 & 6.5 & 8 & 4 & 6 \\
\hline & $\mathrm{Mg}$ XI & 9.23 & 6.5 & 3 & - & - \\
\hline & Mg XI & 9.17 & 6.5 & 16 & 8 & 14 \\
\hline & Cont. & & & 47 & 49 & 22 \\
\hline \multirow[t]{5}{*}{ Be_thick } & Si XIV & 6.19 & 7.1 & - & 5 & 5 \\
\hline & Si XIV & 6.18 & 7.1 & - & 10 & 9 \\
\hline & Si XIII & 6.74 & 6.7 & 6 & - & 8 \\
\hline & Si XIII & 6.65 & 6.7 & 14 & 6 & 20 \\
\hline & Cont. & & & 52 & 60 & 30 \\
\hline
\end{tabular}

Notes. For each filter lines are included in the table only if they contribute more than $3 \%$ to the total count rate for that filter.

above $\log T=6.5 \mathrm{~K}$. The predicted XRT count rates obtained using this DEM curve are included in Table 5. The reduction in the predicted count rates is significant for all channels ranging from $53 \%$ for the C_poly channel to $95 \%$ for the Be_thick channel.

Table 7 contains the predicted fractional contribution of emission lines, as well as continuum emission, to each of the XRT filters for both the active region and quiet Sun areas. For each filter, lines are included in the table only if they contribute more than $3 \%$ to the total count rate for that filter. The combined contribution from lines which individually contribute less than $3 \%$ is significant due to the large number of such lines. For example with the C_poly filter for the active region area $49 \%$ of the total count rate comes from lines which individually contribute less than 3\%. For the active region case (Table 7) the photospheric 2 abundances of Asplund et al. (2009) are used. For the quiet Sun case (Table 7) the same abundances are used but with the oxygen and neon abundances reduced by a factor of 3 (coronal 2).

\subsubsection{Flare}

Synthetic spectra have been calculated from the EIS flare DEMs corresponding most closely in time to the XRT observations, using CHIANTI. For each of the flare cases the synthetic spectrum for each filter was integrated in order to predict the total count rate. The predicted count rates are included in Table 6.

Table 7 contains the predicted fractional contribution of emission lines, as well as continuum emission, to each of the XRT filters for the flare case. Again for each filter, lines are included in the table only if they contribute more than $3 \%$ to the total count rate for that filter. For the flare case (Table 7) the coronal 1 abundances of Feldman et al. (1992) are used.

\section{Discussion of results}

\subsection{XRT count rates}

For the active region area (Tables 2 and 3) we find excellent agreement between the observed and predicted XRT count rates when using coronal 1 abundances. Similar results are obtained assuming coronal 2 abundances. Setting to zero the hightemperature component does not have a large effect.

For the quiet Sun area (Tables 4 and 5) the observed XRT count rates are also in closer agreement with the predicted count rates using coronal 1 abundances. However, for some of the filters, even with coronal abundances, there is a significant disagreement between the observed and predicted values. This is due to the very low count rates.

\subsection{Spectral contributions to the XRT channels}

For the active region (see Fig. 6) and quiet Sun cases with the Al_poly, C_poly, Ti_poly and Be_thin filters, as well as the filter combination Al_poly-Ti_poly, the most significant contribution comes from Fe XVII lines, with smaller contributions from O VII, O VIII, Ne IX, Ne X, Mg XI and Fe XVIII lines, as well as continuum emission. For the same filters in the flare cases, there are significant contributions from Fe XVII, Fe XVIII, Fe XIX, Fe XX, Fe XXI and Fe XXII lines, as well as continuum emission. With the Be_med filter for both the active region (see Fig. 7) and quiet Sun cases the most significant contribution comes from continuum emission, with smaller contributions from $\mathrm{Mg}$ XI and Fe XVII lines. For the flare case the most significant contribution to the Be_med filter comes from $\mathrm{Mg}$ XI, 
B. O'Dwyer et al.: Response of Hinode XRT to quiet Sun, active region and flare plasma
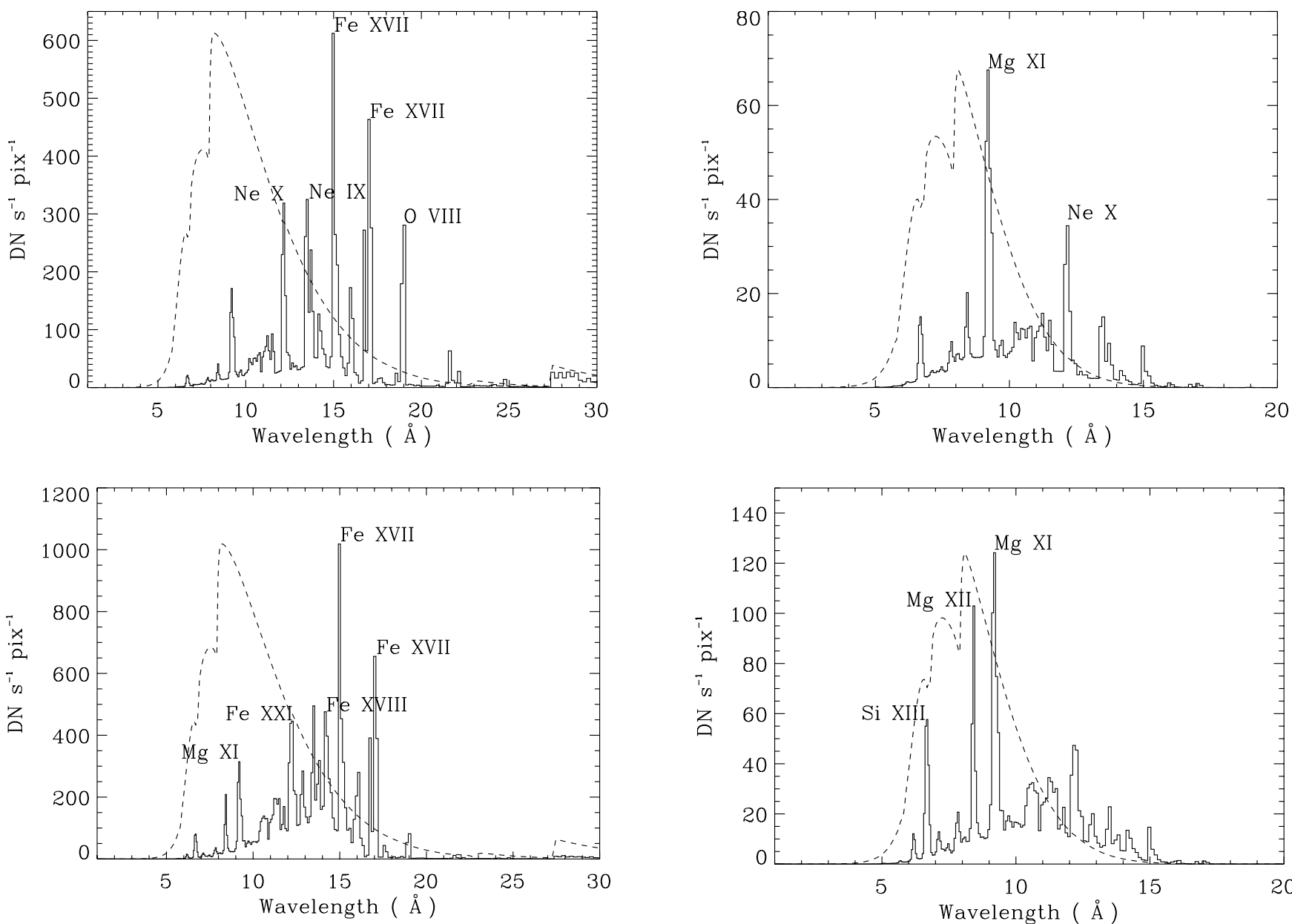

Fig. 6. Top: active region synthetic spectrum for the Ti_poly filter using the photospheric abundances of Asplund et al. (2009). Bottom: flare synthetic spectrum for the Ti_poly filter using the coronal abundances of Feldman et al. (1992) and the "Flare 2" observation. The effective area is overplotted as a dashed line (scaled). The peak intensities of stronger lines are indicated.

Mg XII, Si XIII, Fe XIX, Fe XX, Fe XXI, and Fe XXII lines, as well as continuum emission. With the Be_thick filter for both the active region (see Fig. 8) and quiet Sun cases the most significant contribution comes from continuum emission, with smaller contributions from Si XIII and Si XIV lines. For the flare case the most significant contribution to the Be thick filter comes from Si XIII and Si XIV lines, as well as continuum emission. With the Al_thick filter for both the active region (see Fig. 9) and quiet Sun cases the most significant contributions come from $\mathrm{Mg}$ XI lines and continuum emission, with a smaller contribution from $\mathrm{Mg}$ XII lines. For the flare case the most significant contribution to the Al_thick filter comes from Mg XI and Mg XII lines, along with continuum emission, with smaller contributions from Fe XXI and Fe XXII lines.

\section{Conclusions}

The agreement we obtain between the observed XRT count rates and those predicted by the EIS DEM curves using the coronal 1 abundance set for the active region area is remarkable, considering the uncertainty of 20-30\% in the EIS calibration (Del Zanna 2013b). It suggests that the XRT calibration is consistent with the new EIS calibration, although the agreement is conditional on the element abundances used. The agreement we obtain

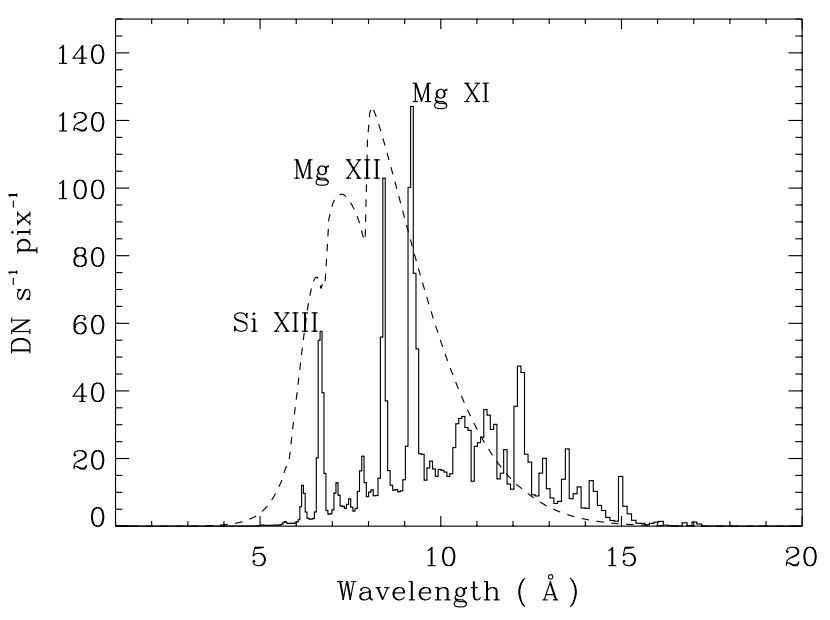

Fig. 7. Same as Fig. 6 for the Be_med filter.

using the DEM modelling also suggests that the CHIANTI v.7.1 atomic data are fairly accurate and complete for the spectral ranges we have considered. The good agreement with a FIP enhancement of a factor of about 4 (coronal 1) is consistent with the active region core abundance results of (Mason 1975) and (Del Zanna 2013a), where a factor of about 3.2 was found. We note that this result (i.e. good agreement between EIS and XRT) is in contrast to that obtained by Testa et al. (2011). The main reason for the different result is due to the new EIS calibration.

For the quiet Sun case the agreement between observed and predicted count rates is also quite good using coronal abundances for those filters which have larger observed count rates (greater than $\sim 18 \mathrm{DN} \mathrm{s}^{-1} \mathrm{pix}^{-1}$ ). However, for the other filters with lower counts there is a substantial disagreement between the observed and predicted count rates. For the quiet Sun case the only constraint we can place on emission from plasma at temperatures larger than $\log T[\mathrm{~K}] \sim 6.5$ comes from upper limits on line intensities. As a result it is quite possible that we are overestimating the predicted contribution of lines formed at these temperatures. As can be seen from Table 7, even in the quiet Sun case, the predicted contribution from lines formed at temperatures larger than $\log T[\mathrm{~K}] \sim 6.5$ represents quite a significant fraction of the total emission for many of the filters. If we set the quiet Sun photospheric 2 EIS DEM curve to zero above $\log T(\mathrm{~K})=6.5$ the predicted count rates are reduced significantly for all channels ranging from $53 \%$ for the C_poly channel to $95 \%$ for the Be_thick channel. 

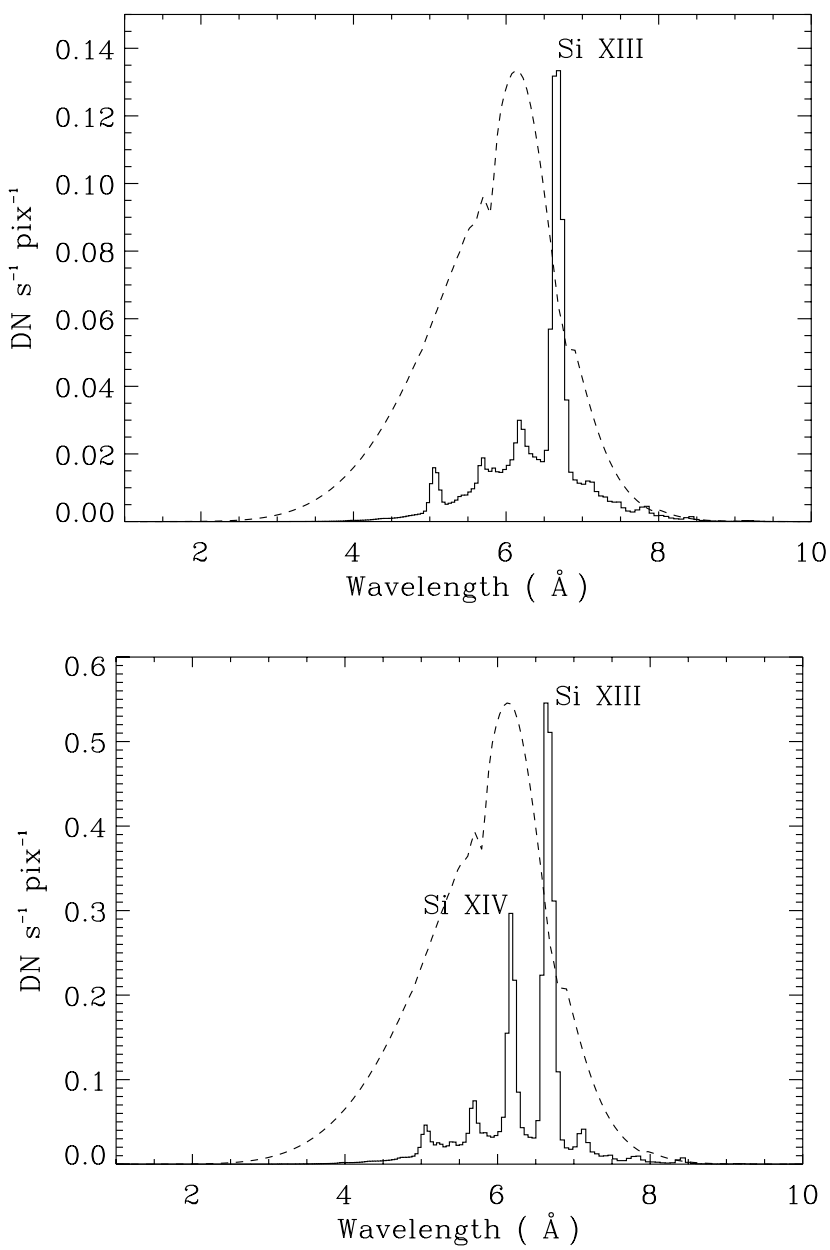

Fig. 8. Same as Fig. 6 for the Be_thick filter.

For the flare cases, good agreement is found for the Be_thick and Ti_poly filters within a wide range of predicted count rates when different abundance datasets are considered. The predicted Al_thick count rates are consistently larger by at least $20 \%$ than the observed values for each of the abundance datasets.

Having investigated both active region and flare observations it is clear that an additional uncertainty in interpreting the XRT observations comes from the unkown elemental abundances. We found that these affect some filters more than others, and not only directly via spectral lines, but also via changes in the continuum primarily due to changes in the abundance values of some high FIP elements.

Using the photospheric abundances of Asplund et al. (2009) rather than those of Grevesse et al. (1998) reduces the predicted intensities of the high-FIP lines, primarily oxygen and neon, and thus the total count rate for each filter. Similarly, the use of coronal rather than photospheric abundances reduces the predicted intensities of oxygen and neon lines and thus the total count rate for each filter by between $20-40 \%$. The continuum, which consists mainly of free-free and free-bound emission, is also reduced by the use of coronal abundances, but only slightly. As a consequence differences in the predicted count rates between the two coronal abundance datasets are within a few percent.

The contribution of spectral lines and continuum emission to individual XRT channels varies quite significantly depending on what type of plasma is being observed. In particular the lines
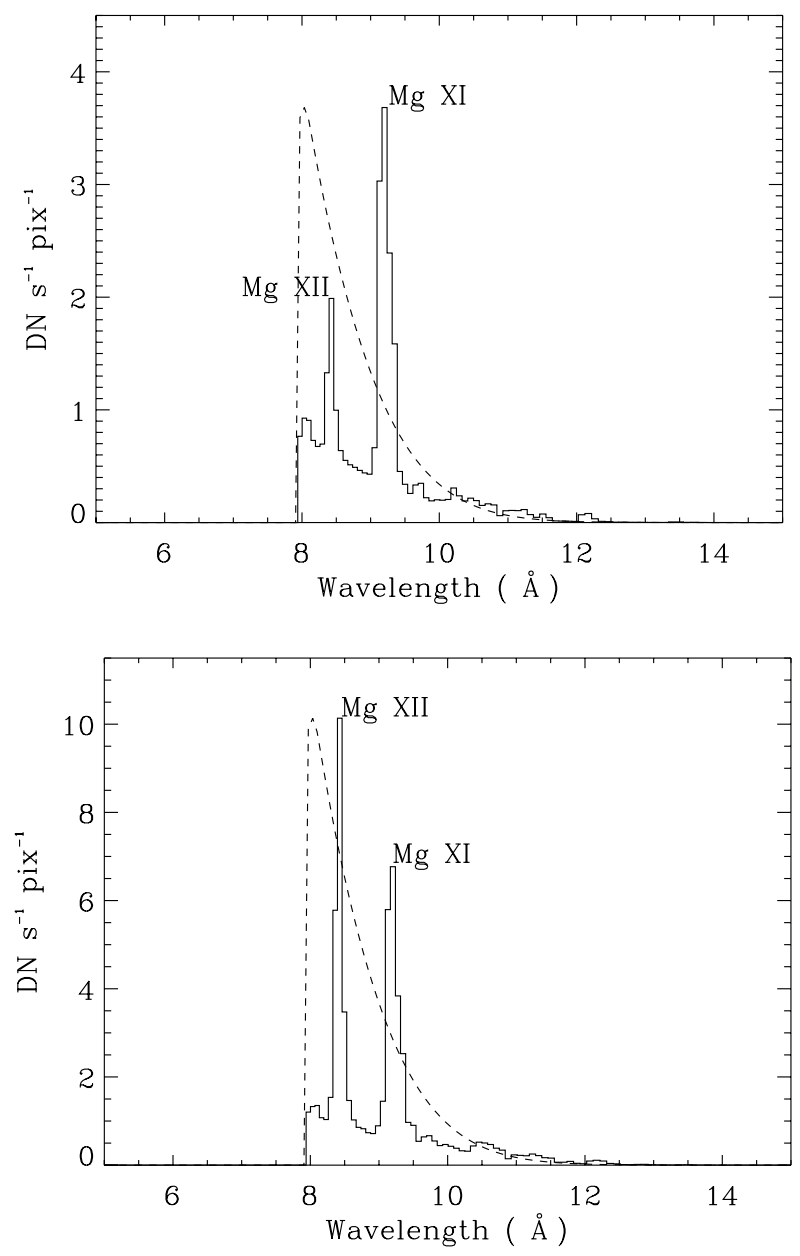

Fig. 9. Same as Fig. 6 for the Al_thick filter.

which contribute for flare plasma can be quite different to those which dominate the XRT channels in active regions.

The calibration of XRT is still evolving. We have used the most recently available (in SSW) calibration, but we are aware that work is in progress (Narukage 2011) on an updated calibration. We understand that the thick XRT filters may be most affected.

Acknowledgements. We acknowledge support from STFC (UK). B.O.D. was supported by funding from the Gates Cambridge Trust. We would like to thank Durgesh Tripathi and Alphonse Sterling for helping with the design of the observing sequences. We would also like to thank Mark Weber for his advice on creating XRT DEM curves. CHIANTI is a collaborative project involving the NRL (USA), the Universities of Florence (Italy) and Cambridge (UK), and George Mason University (USA). Hinode is a Japanese mission developed and launched by ISAS/JAXA, collaborating with NAOJ as a domestic partner, NASA and STFC (UK) as international partners. Scientific operation of the Hinode mission is conducted by the Hinode science team organized at ISAS/JAXA. This team mainly consists of scientists from institutes in the partner countries. Support for the post-launch operation is provided by JAXA and NAOJ (Japan), STFC (UK), NASA, ESA, and NSC (Norway).

\section{References}

Asplund, M., Grevesse, N., Sauval, A. J., \& Scott, P. 2009, ARA\&A, 47, 481 Bradshaw, S. J., \& Raymond, J. 2013, Space Sci. Rev., 271

Culhane, J. L., Harra, L. K., James, A. M., et al. 2007, Sol. Phys., 243, 19

Del Zanna, G. 2009, A\&A, 508, 501

Del Zanna, G. 2013a, A\&A, 558, A73 
B. O'Dwyer et al.: Response of Hinode XRT to quiet Sun, active region and flare plasma

Del Zanna, G. 2013b, A\&A, 555, A47

Del Zanna, G., \& Ishikawa, Y. 2009, A\&A, 508, 1517

Del Zanna, G., O’Dwyer, B., \& Mason, H. E. 2011, A\&A, 535, A46

Dere, K. P., Landi, E., Mason, H. E., Monsignori Fossi, B. C., \& Young, P. R. 1997, A\&AS, 125, 149

Dere, K. P., Landi, E., Young, P. R., et al. 2009, A\&A, 498, 915

Feldman, U., Mandelbaum, P., Seely, J. F., Doschek, G. A., \& Gursky, H. 1992, ApJS, 81, 387

Feldman, U., Schühle, U., Widing, K. G., \& Laming, J. M. 1998, ApJ, 505, 999

Fludra, A., \& Schmelz, J. T. 1995, ApJ, 447, 936

Freeland, S. L., \& Handy, B. N. 1998, Sol. Phys., 182, 497

Golub, L., Deluca, E., Austin, G., et al. 2007, Sol. Phys., 243, 63

Grevesse, N., \& Sauval, A. J. 1998, Space Sci. Rev., 85, 161

Haugan, S. V. H. 1997, The Component Fitting System (CFIT) for IDL, CDS software note no. 47, solar.bnsc.rl.ac.uk/software/notes.shtml

Kashyap, V., \& Drake, J. J. 1998, ApJ, 503, 450

Kosugi, T., Matsuzaki, K., Sakao, T., et al. 2007, Sol. Phys., 243, 3
Landi, E., Del Zanna, G., Young, P. R., Dere, K. P., \& Mason, H. E. 2012, ApJ, 744, 99

Landi, E., Young, P. R., Dere, K. P., Del Zanna, G., \& Mason, H. E. 2013, ApJ, 763,86

Lang, J., Kent, B. J., Paustian, W., et al. 2006, Appl. Opt., 45, 8689

Lemen, J. R., Bentley, R. D., \& Sylwester, J. 1986, Adv. Space Res., 6, 245

Mason, H. E. 1975, MNRAS, 171, 119

Narukage, N. 2011, Hinode 5 poster

Narukage, N., Sakao, T., Kano, R., et al. 2011, Sol. Phys., 269, 169

O’Dwyer, B., Del Zanna, G., Mason, H. E., et al. 2011, A\&A, 525, A137

Reale, F., Testa, P., Klimchuk, J. A., \& Parenti, S. 2009, ApJ, 698, 756

Schmelz, J. T., Saar, S. H., DeLuca, E. E., et al. 2009, ApJ, 693, L131

Schmelz, J. T., Reames, D. V., von Steiger, R., \& Basu, S. 2012, ApJ, 755, 33

Testa, P., Reale, F., Landi, E., DeLuca, E. E., \& Kashyap, V. 2011, ApJ, 728, 30

Veck, N. J., \& Parkinson, J. H. 1981, MNRAS, 197, 41

Wang, T., Thomas, R. J., Brosius, J. W., et al. 2011, ApJS, 197, 32

Witthoeft, M. C., \& Badnell, N. R. 2008, A\&A, 481, 543 